\title{
Determination of XUV Optical Constants by Reflectometry Using a High-Repetition Rate 46.9-nm Laser
}

\author{
I. A. Artioukov, B. R. Benware, J. J. Rocca, M. Forsythe, Yu. A. Uspenskii, and A. V. Vinogradov
}

\begin{abstract}
We report the measurement of the optical constants of $\mathrm{Si}$, GaP, InP, GaAs, GaAsP, and Ir at a wavelength of 46.9 $\mathrm{nm}(26.5 \mathrm{eV})$. The optical constants were obtained from the measurement of the variation of the reflectivity as a function of angle utilizing, as an illumination source, a discharge pumped 46.9. nm table-top laser operated at a repetition rate of $1 \mathrm{~Hz}$. These measurements constitute the first application of an ultrashort wavelength laser to materials research.
\end{abstract}

Index Terms-Soft X-ray laser, XUV optical constants, XUV reflectometry.

\section{INTRODUCTION}

$\mathbf{T}$ HE GROWING interest that we are presently witnessing in the optical properties of solids in the wavelength interval of $35-50 \mathrm{~nm}$ is largely stimulated by the advent of compact lasers and high-order harmonic sources at these wavelengths [1]-[4]. Another reason is the development of efficient multilayer optics at these wavelengths [5], [6] for use in synchrotron radiation research [7], [8] and solar physics [9]. However, the progress in this area depends significantly on the knowledge of the optical constants of materials within this wavelength range. For some materials, the optical constants at XUV wavelengths have been tabulated [10]. However, for many solids the only available data are those from atomic scattering factors [11], which ignore the effects of interatomic bonding on the optical spectra. The data show that most of the elements, among them oxygen, nitrogen, carbon and fluorine, are highly absorbing in the interval under consideration. The fact that these materials usually form thin surface contamination layers greatly complicates the accurate measurement of optical constants, especially for the materials with low absorption.

The most popular method for the determination of optical constants is the measurement of normal incidence reflectiv-

Manuscript received May 17, 1999. This work has been performed in frames of the CRDF under Project RP1-240. The development of the laser was supported by the National Science Foundation under Grant DMR-9512282. The work of Yu. A. Uspenskii was supported by the Russian Federal Program "Integration," under Grant K0 573. The work of B. R. Benware, J. J. Rocca, and M. Forsythe was supported by the Colorado Advance Technology Institute.

I. A. Artioukov, Yu. A. Uspenskii, and A. V. Vinogradov are with the P. N. Lebedev Physical Institute, Moscow 117924, Russia.

B. R. Benware, J. J. Rocca, and M. Forsythe are with the Colorado State University, Electrical Engineering Department, Fort Collins, CO 80523 USA. Publisher Item Identifier S 1077-260X(99)09895-0. ity in a wide spectral interval and the application of the Kramers-Kronig relation to calculate both the refractive and absorption indexes [12], [13]. To do this evaluation one should assume that the reflection of the material is described by the Fresnel formulas. This method results in precise optical constants over the entire wavelength interval, provided the measurement interval is wide enough and the sample surface is free from surface layer impurities. However, most materials develop surface layers of oxide and other impurities when exposed to a natural atmospheric environment. These surface layers can introduce significant errors in the determination of the optical constants for the bulk material.

A second method for the determination of optical constants employs the measurement of angular dependent reflectivity. In this method optical constants at each wavelength can be found by fitting the measured angular dependence of the reflectivity with the Fresnel formula [14]. A deviation of the experimental data from the calculated curve for the bulk material is a signal that there is an additional contribution to the reflectivity, which is most likely due to an oxide surface layer. Thus, this method can in principle provide not only optical constants of the bulk material but also information about the surface characteristics of the sample. Moreover, as illustrated herein, when the latter is optically thin, the reflection from the bulk material can be separated from the reflection caused by the surface layer. Therefore, the optical constants of solids can be determined even on partially oxidized or contaminated samples.

We have developed experimental and calculation procedures for the determination of optical constants at the XUV wavelengths using angular dependent laser reflectometry. The key device that made the measurements possible is a high repetition rate table-top soft X-ray laser operating at a wavelength of $46.9 \mathrm{~nm}$ [2]. The laser delivers a beam of $26.5 \mathrm{eV}$ with an average power of up to $1 \mathrm{~mW}$ within a spectral bandwidth of $\lambda / \Delta \lambda>10^{4}$. Section II describes the experimental setup used in the reflectivity measurements. Section III discusses the models of a sample's surface used to fit the reflectivity data and gives the details of the data processing. Section IV presents the results of our evaluation of optical constants for $\mathrm{Si}, \mathrm{GaP}, \mathrm{InP}$, GaAs, GaAsP, and Ir, and gives a comparison with previously published optical constants. Based on these data, we analyze the sensitivity of the procedure to the purity of the sample's surface. Finally, in the last section we discuss the features and prospects for the determination of optical constants in the XUV with the help of ultrashort wavelength lasers. 


\section{EXPERIMENTAL SETUP}

The experimental setup used in the measurements of the reflectance is shown in Fig. 1. The samples were illuminated with the beam of a Ne-like Ar capillary discharge laser operating in a single line at a wavelength of $46.9 \mathrm{~nm}$. The characteristics of this table-top soft X-ray laser were described in a previous publication [2]. In this laser, the gain medium is an elongated plasma column generated in a capillary channel by a fast discharge current pulse. The magnetic force of the current pulse rapidly compresses the plasma to form a dense and hot column with length to diameter aspect ratio approaching 1000:1, where the amplification is obtained following collisional excitation of the $3 \mathrm{~s}{ }^{1} \mathrm{P}_{1}^{0}-3 \mathrm{p}{ }^{1} \mathrm{P}_{0}$ transition in $\mathrm{Ne}$ like $\mathrm{Ar}$ ions [1], [2], [15]. For this experiment, laser pulses with an energy of approximately $0.1 \mathrm{~mJ}$ and a pulsewidth of $\approx 1.2 \mathrm{~ns}$ were generated at a repetition rate of $1 \mathrm{~Hz}$. The laser beam divergence was approximately $4.5 \mathrm{mrad}$.

The measurements were conducted in a vacuum chamber placed at about $1.5 \mathrm{~m}$ from the exit of the laser. The samples were mounted on the axis of a rotational stage driven by a stepper motor, which allowed for the selection of angles of incidence between $0^{\circ}$ and $90^{\circ}$. The intensity of the reflected beam was recorded with a vacuum photodiode (labeled "A" in Fig. 1), that was mounted on a lever arm that followed the angular motion of the reflected beam. A 1-mm-diameter pinhole was placed at the entrance of the chamber to reduce the spot size of the laser beam incident on the sample, which allowed for measurements at grazing angles approaching zero degrees. To overcome scattering of the data due to shot-to-shot intensity variation of the laser, the intensity of the reflected beam was normalized by the intensity of the incident beam for each laser pulse. For this purpose a reference beam was generated by placing a 50\% transmissive gold-plated grid in the path of the incident beam. The intensity of the reference beam reflected by the grid was measured by a second fixed vacuum photodiode (labeled "B" in Fig. 1), and used for the normalization. To obtain absolute reflectance measurements, the signal of the reference photodiode was calibrated with respect to the intensity of the beam transmitted by the grid by removing the sample and positioning the rotating diode in the beam path. This calibration was determined with an error of less than $0.5 \%$. The angular dependence of the reflectivity was measured by scanning the angle of incidence while repetitively firing the laser at a repetition frequency of $1 \mathrm{~Hz}$. The photodiode signals corresponding to the intensity of the reflected beam and reference beams were recorded and stored for every laser shot by a $500-\mathrm{MHz}$ digitizing oscilloscope (Hewlett-Packard model 54 825A).

Fig. 2 is an example of the reflectance data obtained. It shows a single measurement run of the reflectivity as a function of incident angle for a sample of polished crystalline $\mathrm{Si}$. This data depicts a typical measurement that consisted of 300 contiguous laser pulses for a $90^{\circ}$ rotation of the sample. At small angles of incidence, photodiode "A" blocks the beam from impinging on the sample limiting the minimum angle at which data could be obtained to $1.6^{\circ}$. This angle, which corresponds to the first valid data point near normal

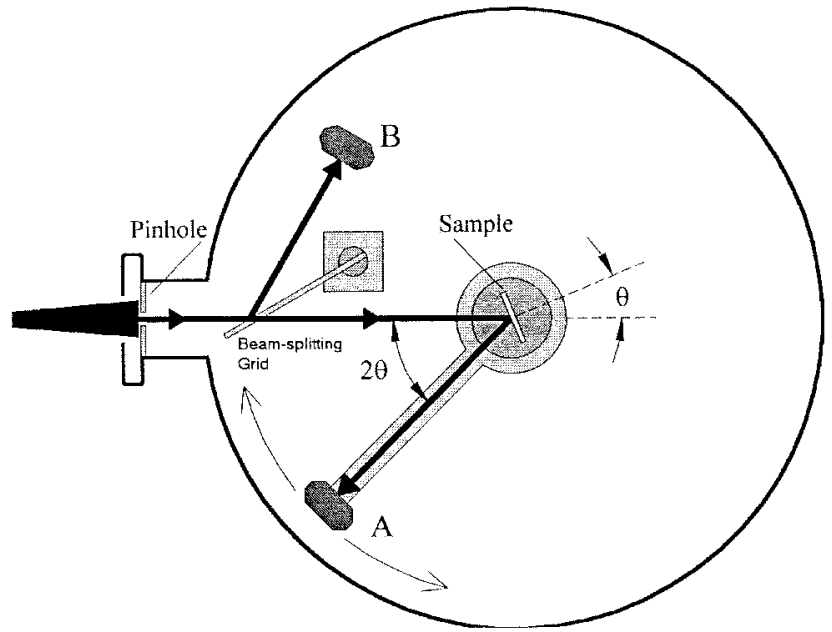

(a)

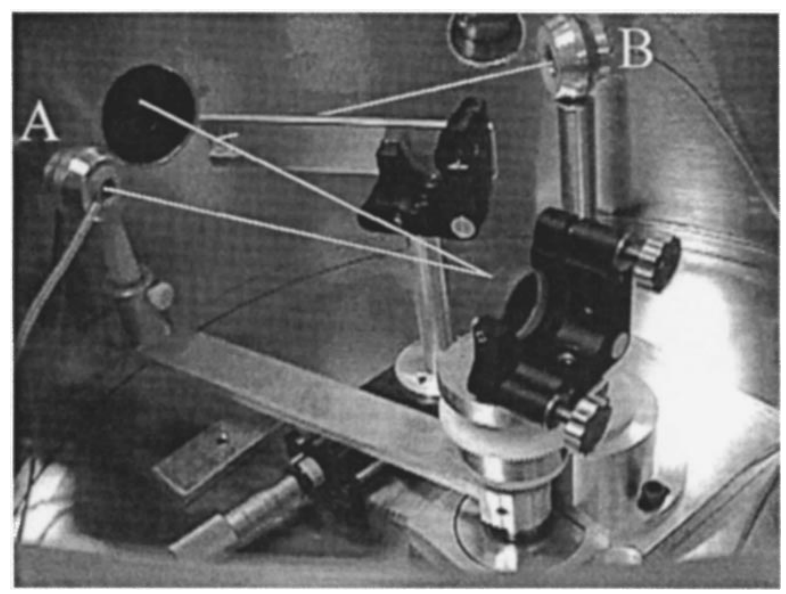

(b)

Fig. 1. Schematic diagram and photograph of the laser reflectometer used in the measurement of XUV optical constants.

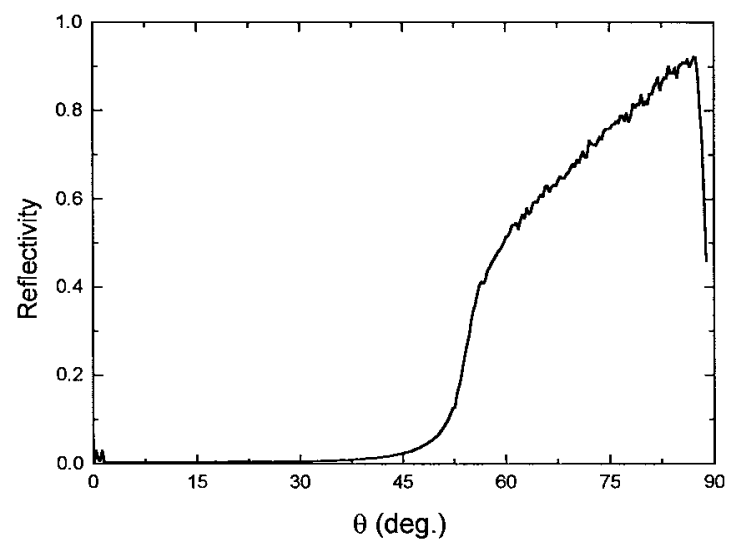

Fig. 2. Example of measured reflectivity vs incident angle dependence in the range $0^{\circ}<\theta<90^{\circ}$ for a $\mathrm{Si}$ sample.

incidence, was determined from the geometric dimensions of the system and was used to relate each data point to its corresponding angle. At the other extreme, as the incident angle approaches $90^{\circ}$, the projection of the incident beam on the sample becomes larger than the sample and therefore limited the maximum angle at which valid data could be 
obtained. In the specific case of the data for the Si sample shown in Fig. 2, the $1 \mathrm{~mm}$ diameter of the beam limited the measurement to angles less than $85.5^{\circ}$. This accounts for the apparent decrease of the reflectivity at grazing angles that should otherwise approach $100 \%$.

\section{DATA PROCESSING}

The correct interpretation of measured reflectivity data substantially depends on an adequate model of a sample surface and on the fitting procedure. Three models of the sample surface were investigated to interpret the data. They, respectively, assume: (a) the sample is homogeneous on the scale of light penetration depth; (b) a homogeneous bulk material with a single layer at the surface; and (c) a bulk material with two different surface layers.

The first model corresponds to a pure material with complex refractive index $\tilde{n}_{b}=n+i \cdot k$ ( $n$ and $k$ are fitting parameters of the model) with an ideally smooth surface. It results in the reflection that is described by the Fresnel formulas. For an $s$-polarized beam the reflection amplitude is of the form:

$$
r_{v b}=\frac{\tilde{n}_{v} \cos \theta-\tilde{n}_{b} \cos \theta_{b}}{\tilde{n}_{v} \cos \theta+\tilde{n}_{b} \cos \theta_{b}}
$$

where $\tilde{n}_{v}=1$ is the refractive index for vacuum, $\theta$ is the incident angle and $\theta_{b}$ is given by Snell's law: $\tilde{n}_{v} \sin \theta=$ $\tilde{n}_{b} \sin \theta_{b}$. For a beam with $p$-polarization the only change is the substitution of $1 / \tilde{n}$ for $\tilde{n}$ in $r_{v b}$.

The second model assumes a sample with a single homogeneous layer at the surface, which has an ideally sharp permittivity profile and smooth interface with the bulk material. The model has five parameters: the complex refractive indexes for the surface layer $\tilde{n}_{l}$ and bulk material $\tilde{n}_{b}$, and the layer thickness $d_{l}$. For this model, the reflection amplitude is given by

$$
r=\frac{r_{v b}+r_{l b} \exp (2 i D)}{1+r_{v l} r_{l b} \exp (2 i D)}
$$

where $D=n_{l} \cos \theta\left(2 \pi d_{l} / \lambda\right)$, and $r_{v l}, r_{l b}$ are the reflection amplitudes at the interfaces: vacuum-surface layer and surface layer-bulk material, correspondingly. These amplitudes are described by equations similar to (1).

The third model imitates a gradual decrease of oxidization from the surface to the bulk material. It contains eight parameters: the complex refractive indexes for the first layer $\tilde{n}_{l 1}$, the second layer $\tilde{n}_{l 2}$, the bulk material $\tilde{n}_{b}$ and the thickness of each layer $d_{l 1}$ and $d_{l 2}$. In this case, the magnitude of the reflection can again be put in the form of (2) with the substitution of $r_{v l 1}$ for $r_{v l 2}$ and $r_{l 1 l 2}$ for $r_{l b}$, where $r_{l 1 l 2}$ is the reflection amplitude at the interface between the first and second layers.

Note that all three models ignore microroughness of the surface and the interfaces. It is known that microroughness mainly redistributes reflected light between specular reflection and diffuse scattering. The aperture of our detector was $8.8 \mathrm{~mm}$ in diameter and sustained an angle of $44 \mathrm{mrad}$ with respect to the beam spot on the sample. Estimation of scattering effects shows that for a roughness correlation length greater than 14 $\mu \mathrm{m}$ the majority of the scattered radiation was registered by the detector, even at grazing angles. For higher spatial frequency roughness the grazing angle scattering was evaluated to be negligible if the root-mean-square (rms) roughness height is less than $0.5 \mu \mathrm{m}$. For this reason the effect of microroughness was not taken into account in our calculations. A detailed discussion of this problem can be found in [16].

The fitting of the model parameters was carried out by the standard least-squares method using the following merit function $F$ :

$$
F=\frac{1}{N} \sum_{i=1}^{N}\left(\left(R_{\exp }\left(\theta_{i}\right)-R_{\mathrm{th}}\left(\theta_{i}\right)\right) / R_{\exp }\left(\theta_{i}\right)\right)^{2}
$$

where $R_{\exp }\left(\theta_{I}\right)$ and $R_{\mathrm{th}}\left(\theta_{i}\right)$ are the measured and calculated reflectivities at the incident angle $\theta_{I}$, and $N$ is the number of angles at which measurements were obtained. Since the measurements were carried out with unpolarized light the calculated reflectivity was averaged over the two polarizations, that is $R_{\mathrm{th}}=\left(R_{\mathrm{th}}^{s}+R_{\mathrm{th}}^{p}\right) / 2$. One can see that the function $F$ is written in the form of the squared relative rms error of the fitting.

To judge the validity of the model, the obtained minimum of $F$ should be compared with the relative rms error of the experiments, which can be estimated as

$$
\langle\Delta R / R\rangle=\sqrt{\frac{1}{N} \sum_{i=1}^{N}\left(\left(R_{\exp }\left(\theta_{i}\right)-\left\langle R_{\exp }\left(\theta_{i}\right)\right\rangle\right) /\left\langle R_{\exp }\left(\theta_{i}\right)\right\rangle\right)^{2}}
$$

where $\left\langle R_{\exp }\left(\theta_{I}\right)\right\rangle$ is the smoothed experimental angular dependent reflectivity. Assuming that the true reflectivity should not oscillate inside the interval of $\Delta \theta \sim 5^{\circ}$, we smoothed the measured reflectivity by the least squares fitting to a quadratic polynomial in $\theta$ over $\Delta \theta$.

The calculations showed that the second model minimizes the merit function $F$ much better than the first. However, the third model only slightly decreases the minimum of $F$ in comparison with the single layer model, and the difference between the merit functions was much less than $\langle\Delta R / R\rangle^{2}$. In other words, the reflectivity was demonstrated to be dependent on the optical thickness of the surface layer, but rather insensitive to its spatial distribution. This fact allowed us to exclude the third model, and thus the final calculations of optical constants described in the next section are based only on the first and the second models. The fitting parameters were found by minimization of the merit function $F$ up to convergence using a quasi-Newton method. This technique allowed us to find the local minimum of $F$ with the correspondent values of the optical constants [17]. The typical time of the computations was $2-4 \mathrm{~s}$ for the first model and $10-30 \mathrm{~s}$ for the second model (Pentium II 233-MHz class computer).

\section{RESUlts OF THE DETERMINATION of OPTICAL CONSTANTS}

Measurements of the dependence of the reflectivity on the angle of incidence were performed using the setup described in Section II. To decrease random experimental errors, several runs of measurements were usually carried out and the results 
were averaged to produce one reflectivity curve. From the experimental reflectivity dependence such as that shown in Fig. 2 we chose a range of validity for the data: $1.8^{\circ}<\theta<85^{\circ}$ (affected by the sample's size), which usually consisted of 270-350 data points.

All the semiconductor samples were bulk crystalline with a 100 orientation with the exception of the GaAsP, which was an epitaxial layer grown on a GaAs wafer of 100 orientation. The Si sample was $\mathrm{P}$ type with B dopant at a carrier concentration of $5 \times 10^{17}\left(\mathrm{~cm}^{-3}\right)$. The GaP was $\mathrm{P}$ type with $\mathrm{Zn}$ dopant at a carrier concentration of $1.2 \times 10^{18}\left(\mathrm{~cm}^{-3}\right)$. The InP was $\mathrm{N}$ type with $\mathrm{S}$ dopant at a carrier concentration of $1 \times 10^{18}$ $\left(\mathrm{cm}^{-3}\right)$. The GaAs was $\mathrm{P}$ type with $\mathrm{Zn}$ dopant at a carrier concentration of $1.2 \times 10^{19}\left(\mathrm{~cm}^{-3}\right)$. The GaAsP sample was a thick epitaxially grown layer of $\mathrm{GaAs}_{0.6} \mathrm{P}_{0.4}$ on a $\mathrm{GaAs}$ substrate. Due to the strain from a lattice mismatch between the epitaxial layer and the substrate, this sample exhibited periodic surface structure. However, the rms roughness of this structure was measured to be less than $0.5 \mu \mathrm{m}$. The Ir was electron beam evaporated as a thick film onto a super polished (RMS surface roughness $\approx 1 \AA$ ) glass substrate.

In order to more fully characterize the influence of the surface layer on the measurements, the samples of $\mathrm{Si}, \mathrm{GaP}$ and InP were chemically treated in the following manner to alter the characteristics of the surface layer. The samples were dipped in a 5\% solution of $\mathrm{HF}$ in distilled water for approximately $5 \mathrm{~min}$ and were then rinsed with acetone and methanol. The samples were exposed to ambient atmospheric conditions for less than $5 \mathrm{~min}$ before being positioned in the system under a vacuum of about $1 \times 10^{-5}$ torr. In the case of the $\mathrm{Si}$, the measured reflectivity for a treated sample was significantly different from that of an untreated sample. However, the reflectivity curve of the treated sample did not appreciably change after exposure to the ambient environment for more than $12 \mathrm{~h}$. For this reason, we believe it is likely that this chemical treatment passivated the Si surface and prevented further oxide growth [18].

The results of fitting the data for each sample with the two models are shown in Figs. 3-8. As an example, let us first consider the data processing technique and evaluation of the optical constants for the Si sample shown in Fig. 3. This figure shows the measured reflectivity of an untreated $\mathrm{Si}$ sample as well as the corresponding results of the least-squares fit. The dotted and solid curves $R(\theta)$ were calculated with the first and the second models, respectively, [(1) and (2) for the reflection amplitudes]. As illustrated in Fig. 3, the shape of the measured reflectivity dependence cannot be fit without assuming the existence of a surface layer. The curve seems to consist of two parts with a kink around $\theta \approx 58^{\circ}$. Despite the fact that each of these parts may be fit by the Fresnel formula (1) it is not possible to obtain a good fit for the entire data set utilizing the first model. However, the second model allows for the fitting of such curves. This fact emphasizes the need to measure the reflectivity over all the angular range including near normal incidence angles where the reflectivity is low. The high intensity of the laser source is an advantage for the accurate measurements of the reflectivity at near-normal incidence, where the reflectivity of most materials is low.

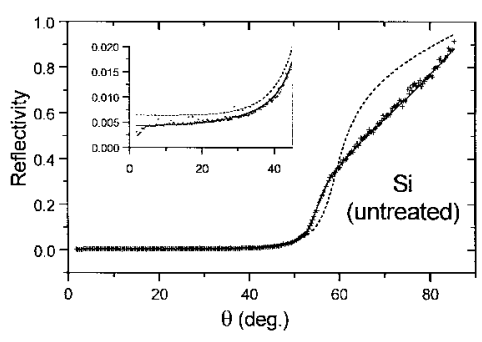

(a)

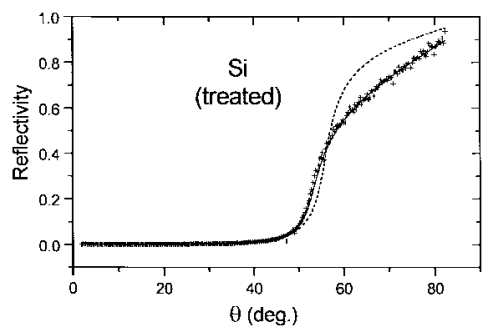

(b)

Fig. 3. Measured and calculated reflectivity for a 100 crystalline Si sample as function of the incident angle $\theta$. (a) Before chemical treatment. The dotted curve is calculated with the optimal optical constants $\tilde{n}_{b}=0.85+i \cdot 0.023$ without a surface layer (the first model). The solid curve was obtained in the frame of the second model with the optimal parameters for $\tilde{n}_{b}=0.82+i \cdot 0.015$ and surface layer parameters: $\tilde{n}_{l}=0.75+i \cdot 0.30$, thickness $d_{l}=1.8 \mathrm{~nm}$; (b) After chemical treatment. The dotted curve corresponds to parameters: $\tilde{n}_{b}=0.85+i \cdot 0.023$ without the surface layer, the solid curve: $\tilde{n}_{b}=0.80+i \cdot 0.021$ (layer: $\tilde{n}_{l}=0.94+i \cdot 0.021$, thickness $d_{l}=9.3 \mathrm{~nm}$ ).

In general the second model provides a much better approximation to the experimental data for all the data obtained. This results in smaller minimum values of the merit function. For example, fitting the data corresponding to the untreated Si sample gives $F_{1}=6 \cdot 10^{-2}$ and $F_{2}=5 \cdot 10^{-3}$ for the first and second models repectively. In addition, note that the minimum of $F_{2}$ is of the order of the experimental RMS error $\langle\Delta R / R\rangle^{2}=2 \cdot 10^{-3}$. It is not surprising that optical constants $n$ and $k$ evaluated in the frames of these two models are different: $n=0.844$ and $k=0.023$ for the first and: $n=0.817$ and $k=0.015$ for the second model. Note that in minimizing $F$ we used permittivities $\varepsilon_{1}=n^{2}-k^{2}$ and $\varepsilon_{2}=2 n k$ as fitting parameters. Though our method is not intended for the accurate determination of the optical constants for the surface layer, the obtained parameters $d_{l}=1.8 \mathrm{~nm}$, $n_{l}=0.75$ and $k_{l}=0.29$ are in this case close to those of a typical layer of $\mathrm{SiO}_{2}$ commonly found on the surface of $\mathrm{Si}$ : $d=2-3 \mathrm{~nm}, n=0.82$ and $k=0.23$ [10]. However, as shown below this is not always the case.

The measured reflectivity of a treated sample of $\mathrm{Si}$ is presented in Fig. 3(b). The measurement was carried out in one run; therefore the scattering of data is larger than in Fig. 3(a). One can observe significant changes in the shape of the angular dependence: the reflectivity is closer to one at grazing angles and the break is smoother. Again, the second model gives a much better description of the experimental dependence. The resulting optical constants of bulk Si are now: $n=0.800$ and $k=0.02$, that is, are only slightly different from those of the same sample of Si before it was chemically treated. This consistency between the results for treated and 


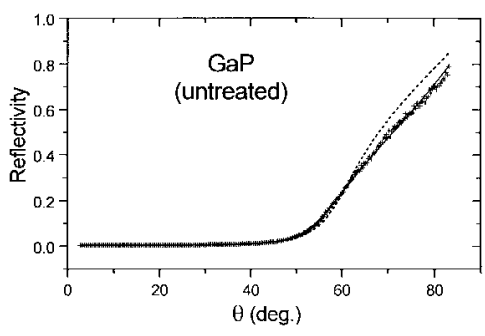

(a)

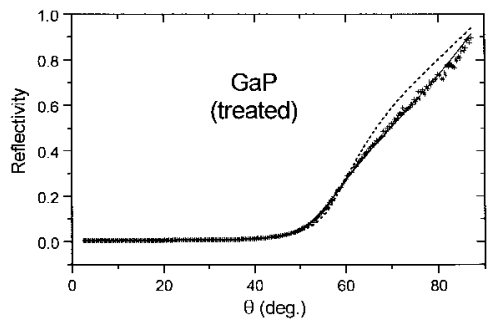

(b)

Fig. 4. Measured and calculated reflectivity for 100 crystalline $\mathrm{GaP}$ as function of the incident angle $\theta$. (a) Before chemical treatment. Dotted curve corresponds to $\tilde{n}_{b}=0.86+i \cdot 0.050$ without the surface layer. Solid curve: $\tilde{n}_{b}=0.82+i \cdot 0.052$ (layer: $\tilde{n}_{l}=0.78+i \cdot 0.44$, thickness $d_{l}=0.98 \mathrm{~nm}$ ). (b) After chemical treatment. The dotted curve corresponds to: $\tilde{n}_{b}=0.85+i \cdot 0.048$ without the surface layer, the solid curve $\tilde{n}_{b}=0.82+i \cdot 0.055$ (layer: $\tilde{n}_{l}=0.82+i \cdot 0.32$, thickness $d_{l}=1.1 \mathrm{~nm}$ ).

untreated samples, which was also found for $\mathrm{GaP}$ and $\mathrm{InP}$, provides evidence of the good accuracy of the method for the determination of optical constants of bulk materials. On the other hand, the best fit to the data of the chemically treated $\mathrm{Si}$ sample gives a rather uncharacteristic value of $9.3 \mathrm{~nm}$ for the thickness of the surface layer. This is an indication that the method is not adequate for the accurate determination of the surface layer parameters.

Figs. 4-7 illustrate the measured reflectivity curves for several other semiconductor materials: GaP, InP, GaAs, GaAsP. All these data show general features similar to that of $\mathrm{Si}$ although, due to higher absorption of these materials, the effects of the absorptive surface layer are less noticeable. For the case of the Ir film (Fig. 8) in which the absorption for the bulk material is the highest, a kink on the angular dependence is not seen and both models give very similar results. Nevertheless, introducing a single surface layer (the second model) leads to a better fit and an improvement in the value of the merit function by several times.

The determined optical constants for the materials that we investigated are collected in Table I along with the available constants from a handbook [10]. The table shows that our values are rather close to those previously reported [10], however, our absorption indexes are lower than those tabulated. This discrepancy may arise from a difference of the chemical and physical properties of the particular samples. While we mostly studied commercially available bulk crystalline samples, the referenced data [10] were obtained for specially prepared materials usually deposited as a thin layer. As was noted in [10], optical constants in UV and XUV spectral range critically depends not only on the chemical composition of the samples but also on a number of technological factors: the temperature

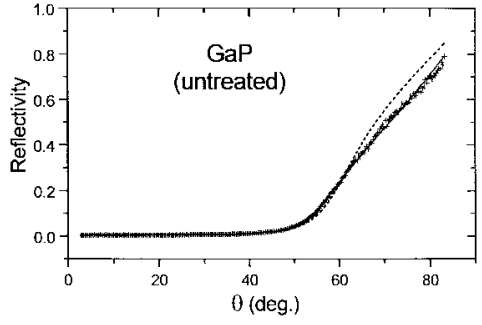

(a)

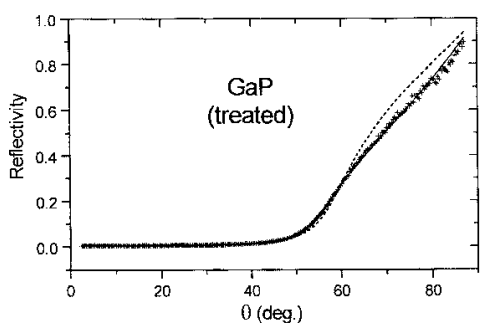

(b)

Fig. 5. Measured and calculated reflectivity for 100 crystalline InP as function of the incident angle $\theta$. (a) Before chemical treatment. The dotted curve corresponds to: $\tilde{n}_{b}=0.92+i \cdot 0.14$ without the surface layer, the solid curve: $\tilde{n}_{b}=0.88+i \cdot 0.087$ (layer: $\tilde{n}_{l}=0.82+i \cdot 0.39$, thickness $d_{l}=1.8 \mathrm{~nm}$ ). (b) After chemical treatment. The dotted curve correponds: $\tilde{n}_{b}=0.91+i \cdot 0.13$ without the surface layer, the solid curve: $\tilde{n}_{b}=0.88+i \cdot 0.09$ (layer: $\tilde{n}_{l}=0.84+i \cdot 0.26$, thickness $d_{l}=2.5 \mathrm{~nm}$ ).

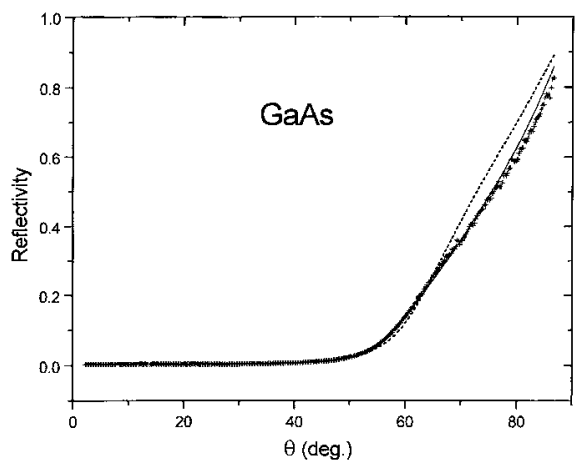

Fig. 6. Measured and calculated reflectivity for 100 crystalline GaAs as function of the incident angle $\theta$. Dotted curve corresponds to: $\tilde{n}_{b}=0.88+i \cdot 0.058$ without the surface layer. Solid curve: $\tilde{n}_{b}=0.84+i \cdot 0.06$ (layer: $\tilde{n}_{l}=0.84+i \cdot 0.30$, thickness $d_{l}=2.4 \mathrm{~nm}$ ).

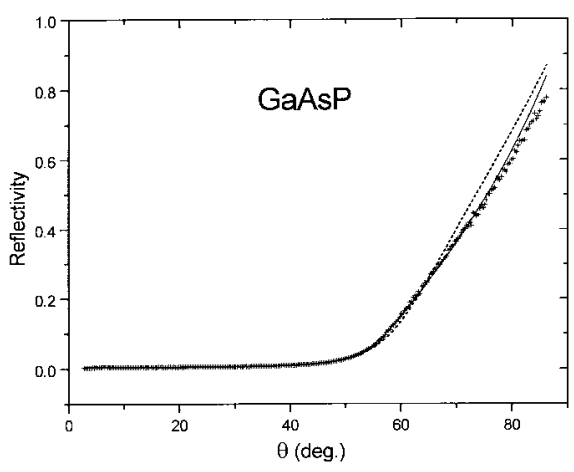

Fig. 7. Measured and calculated reflectivity for a thick epitaxial film of GaAsP as function of the incident angle $\theta$. Dotted curve corresponds to: $\tilde{n}_{b}=0.88+i \quad 0.07$ without the surface layer. Solid curve: $\tilde{n}_{b}=0.83+i \cdot 0.059$ (layer: $\tilde{n}_{l}=0.81+i \cdot 0.39$, thickness $d_{l}=2.0 \mathrm{~nm}$ ). 
TABLE I

Summary of the Optical Constants at 26.5 eV Measured in this Work Using XUV Reflectometry. Previously Measured Values from [10] are also Listed

\begin{tabular}{|c|c|c|c|c|c|c|}
\hline \multirow[t]{2}{*}{ No } & \multirow[t]{2}{*}{ Sample } & \multirow[t]{2}{*}{ Treated } & \multicolumn{2}{|c|}{ This work } & \multicolumn{2}{|c|}{ Ref.[10] } \\
\hline & & & $\mathrm{n}$ & $\mathrm{k}$ & $\mathrm{n}$ & $\mathrm{k}$ \\
\hline$I$ & $\overline{\text { Si }}$ & No & 0.82 & 0.015 & 0.803 & 0.0178 \\
\hline 2 & & Yes & 0.80 & 0.021 & & \\
\hline 3 & GaP & No & 0.82 & 0.052 & N/A & 0.100 \\
\hline 4 & & Yes & 0.82 & 0.055 & & \\
\hline 5 & InP & No & 0.88 & 0.087 & N/A & N/A \\
\hline 6 & & Yes & 0.89 & 0.090 & & \\
\hline 7 & GaAs & No & 0.84 & 0.060 & N/A & 0.083 \\
\hline 8 & GaAsP & No & 0.83 & 0.059 & & \\
\hline 9 & Ir & No & 0.81 & 0.53 & 0.67 & 0.69 \\
\hline
\end{tabular}

N/A: not available.

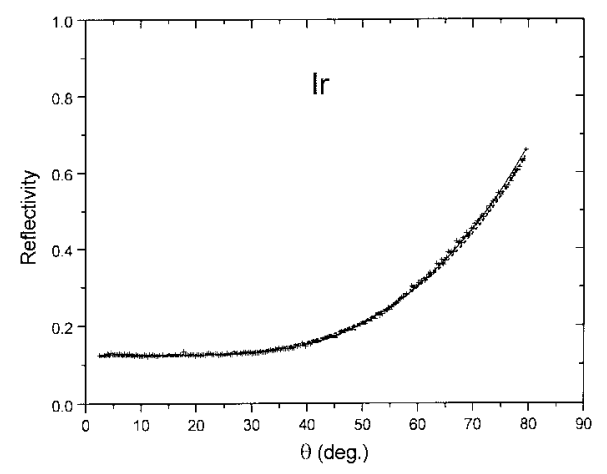

Fig. 8. Measured and calculated reflectivity for an electron beam evaporated Ir film as function of the incident angle $\theta$. Dotted curve corresponds to: $\tilde{n}_{b}=0.76+i \quad 0.60$ without the surface layer. Solid curve: $\tilde{n}_{b}=0.81+i \cdot 0.53$ (layer: $\tilde{n}_{l}=0.83+i \cdot 0.006$, thickness $d_{l}=3.7 \mathrm{~nm}$ ).

of deposition, speed of deposition, vacuum conditions, etc. For example, in the case of $\mathrm{Si}$ (deposited as a thin film), handbook [10] gives two values for $n$ and $k$ obtained for two different samples by two different groups of authors. The two values of the absorption coefficient $k$ differ by about $400 \%$. This example indicates the strong dependency of the optical properties of the samples on particular chemical and physical conditions of manufacturing.

\section{SUMMARY AND DISCUSSION}

Optical constants at a wavelength of $46.9 \mathrm{~nm}$ were determined for $\mathrm{Si}, \mathrm{InP}, \mathrm{GaP}, \mathrm{GaAs}, \mathrm{GaAsP}$, and Ir utilizing a high repetition rate table-top laser emitting at this wavelength. The high intensity of the laser source is an advantage for the accurate measurements of the reflectivity at near-normal incidence, where the reflectivity of most materials is low. The measurements for InP and GaAsP constitute to the best of our knowledge the first experimental value at this wavelength while the rest are in most cases relatively close to those previously tabulated. An important feature of this wavelength region is the very high sensitivity of reflectance to the presence of organic and inorganic compounds, which contaminate the surface of the sample in natural environment conditions. Therefore, to obtain accurate values of optical constants it is important to treat the reflection from the contaminated surface layer on the same footing as the reflection from the bulk material. Our analysis of the data made use of models that take into account the presence of a surface layer of contaminants. Comparison of the values of the optical constants obtained from having surface layers of different characteristics shows that the optical constants of the bulk material obtained with this treatment remain unchanged even when the characteristics of the surface layer changes significantly. This suggests that the approach used in this work might be capable of improving the determination of optical constants, which is important for the design and construction of improved normal incidence reflective coatings for optical devices operating in the XUV. For further work, the separate use of $s$ - and $p$-polarized beams for reflectivity measurements also looks promising as it gives useful additional information. The experiment reported herein constitutes the first application of a soft X-ray laser to materials research. These measurements demonstrate that compact high repetition rate soft X-ray lasers can provide an attractive tabletop alternative to synchrotron sources for certain material science problems.

\section{ACKNOWLEDGMENT}

The authors are indebted to R. M. Feschtchenko and C. Wilmsen for helpful discussions, and to C. D. Macchietto and B. McHardy for their assistance with the experiment. We also thank G. Robinson and C. Menoni for providing some of the samples.

\section{REFERENCES}

[1] J. J. Rocca, V. N. Shlyaptsev, F. G. Tomasel, O. D. Cortazar, D. Hartshom, and J. L. A. Chilla, "Demonstration of a discharge pumped table-top soft X-ray laser," Phys. Rev. Lett., vol. 73, pp. 2192-2195, 1994.

[2] B. R. Benware, C. D. Macchietto, C. H. Moreno, and J. J. Rocca, "Demonstration of a high average power table-top soft X-ray laser," Phys. Rev. Lett., vol. 81, pp. 5804-5807, 1998.

[3] P. V. Nickles, V. N. Shlyptsev, M. Kalashnikov, M. Schnurer, I. Will, and W. Sandner, "Short pulse X-ray laser at $32.6 \mathrm{~nm}$ based on transient gain in Ne-like titanium," Phys. Rev. Lett., vol. 78, pp. 2748-2751, 1997.

[4] A. Rundquist, C. G. Durfee, III, Z. Chang, C. Herne, S. Backus, M. M. Murnane, and H. C. Kapteyn, "Phase-matched generation of coherent soft X-rays," Science, vol. 280, pp. 1412-1415, 1998.

[5] Yu. A. Uspenskii, S. V. Antonov; V. Yu. Fedotov, and A. V. Vinogradov, "Optical properties of 3d-transition metals in the spectral interval of interest for discharged pumped XUV lasers," Proc. SPIE, vol. 3256, pp. 288-294, 1997

[6] Yu. A. Uspenskii, V. E. Levashov, A. V. Vinogradov, A. I. Fedorenko, V. V. Kondratenko, Yu. P. Pershin, E. N. Zubarev, and V. Yu. Fedotov, "High-reflectivity multilayer mirrors for a vacuum-ultraviolet interval of 35-50 nm," Opt. Lett., vol. 23, no. 10, pp. 771-773, 1998. 
[7] D. T. Attwood, P. Naulleau, K. A. Goldberg, E. Tejnil, C. Chang, R. Beguiristain, P. Batson, J. Bokor, E. M. Gullikson, M. Koike, H. Medecki, and J. H. Underwood, "Tunable coherent radiation in the soft X-ray and extreme ultraviolet spectral regions," IEEE J. Quantum Electron., vol. 35, pp. 709-720, May 1999.

[8] H. Zhang, A. Fohlisch, C. Kunz, A. Moewes, M. Pretorius, A. Ranck, H. Sievers, I. Storjohann, V. Wedemeier, and J. Voss, "Optical luminescence spectroscopy with the scanning soft X-ray microscope at HASYLAB/DESY," Rev. Sci. Instrum., vol. 66, no. 2, pp. 2021-2029, 1995.

[9] U. Feldman, "Elemental abundances in the upper solar atmosphere," Physica Scripta, vol. 46, no. 3, pp. 202-220, 1992.

[10] E. D. Palik, Handbook of Optical Constants of Solids. San Diego, CA: Academic, 1998.

[11] B. L. Henke, P. Lee, J. Tanaka, R. L. Simabukuro, and B. K. Fujikawa, "Low-energy X-ray interaction coefficients: Photoabsorption, scattering and reflection," Atom. Data Nucl. Data Tables, vol. 27, pp. 1-144, 1982.

[12] H. R. Phillip, and H. Ehrenreich, "Optical properties of semiconductors," Phys. Rev., vol. 129, no. 4, pp. 1550-1560, 1963.

[13] H. R. Phillip, "Influence of oxide layers on the determination of the optical properties of silicon," J. Appl. Phys., vol. 43, no. 6, pp. $2835-2839,1972$

[14] R. Soufli and E. Gullikson, "Reflectance measurements on clean surfaces for determination of optical constants of silicon in the extreme ultraviolet-soft-X-ray region,” Appl. Opt., vol. 36, pp. 5499-5507, 1997.

[15] J. J. Rocca, D. P. Clark, J. L. A. Chilla, and V. N. Shlyaptsev, "Energy extraction and achievement of the saturation limit in a dischargepumped table-top soft X-ray amplifier," Phys. Rev. Lett., vol. 77, no. 8, 1476-1479, 1996

[16] I. V. Kozhevnikov, V. E. Asadchikov, B. M. Alaudinov, A. Yu. Karabekov, and A. V. Vinogradov, "X-ray investigation of supersmooth surfaces," Proc. SPIE, vol. 2453, pp. 141-153, 1995.

[17] D. Y. Smith, "Dispersion theory, sum rules, and their application to the analysis of optical data," in Hanbook of Optical Constants of Solid, E. D. Palik, Ed. Orlando, FL: Academic Press, 1985, pp. 35-64.

[18] T. Takahagi, I. Nagai, A. Ishitani, H. Kuroda, and Y. Nagasawa, "The formation of hydrogen passivated silicon single-crystal surfaces using ultraviolet cleaning and HF etching," J. Appl. Phys., vol. 64, no. 7, pp. $3515-3521,1988$

[19] I. I. Samoilenko, B. M. Shchedrin, and L. Feigin, "Global minimization in reconstruction of the scattering-length-density profile by X-ray reflectivity data," Phys. B, vol. 221, pp. 542-546, 1996.

[20] H. Tsunematsu, M. Aita, A. Tanaka et. al., "RHEED observation and ARUPS study of Ni deposited on Mo (110) surface," in Abstracts of the 12th Int. Conference on XUV Radiation Physics, San Francisco, CA, Aug. 3-7, 1998

[21] D. Gador, C. Buchberger, R. Fink, and E. Umbach, "Manipulation of molecular orientation in ultrathin organic films: NTCDA on $\operatorname{Ag}(111)$," Europhys. Lett., vol. 41, no. 2, pp. 231-236, 1998.

[22] C. M. Quintella, G. G. B. de Souza, and M. S. P.Mundin, "Ionic fragmentation of CF4 by synchrotron radiation and laser multiphoton ionization," Abstracts of the 12th Int. Conference on XUV Radiation Physics, San Francisco, CA, Aug. 3-7, 1998.

I. A. Artioukov, photograph and biography not available at the time of publication.

B. R. Benware, photograph and biography not available at the time of publication.

J. J. Rocca, photograph and biography not available at the time of publication.

M. Forsythe, photograph and biography not available at the time of publication.

Yu. A. Uspenskii, photograph and biography not available at the time of publication.

A. V. Vinogradov, photograph and biography not available at the time of publication. 